\title{
Fringe Tracker for the VLTI Spectro-Imager
}

\author{
M. Gai ${ }^{1}$, D. F. Buscher ${ }^{2}$, L. Corcione ${ }^{1}$, S. Ligori ${ }^{1}$ and J. S. Young ${ }^{2}$ \\ ${ }^{1}$ Istituto Nazionale di Astrofisica - Osservatorio Astronomico di Torino, Italy \\ email: gai@oato.inaf.it \\ ${ }^{2}$ Astrophysics Group, Cavendish Laboratory, University of Cambridge, UK \\ email: dfb@mrao.cam.ac.uk
}

\begin{abstract}
Interferometry in the near IR aims at providing imaging resolution on the mas scale, and astrometry at the few $\mu a s$ level, from ground based infrastructures using current telescope technology. To take advantage of simultaneous combination of four to eight telescopes, an international consortium is proposing to ESO the development of the VLTI Spectro-Imager. One of the key sub-systems, to measure and correct the atmospheric perturbations relative to the beam phase, is the fringe tracker, aimed at providing the science combiner with long, stable observing conditions. The fringe tracker function in interferometer is equivalent to adaptive optics for conventional telescopes. The fringe tracker concept under study, using minimum redundancy combination and bulk optics, is described.
\end{abstract}

Keywords. instrumentation: interferometers, techniques: interferometric

\section{Introduction}

Several major national and international astronomical facilities for interferometry have been, and are being, built around the world. The goal is to improve on angular resolution and/or measurement precision with respect to individual large telescopes: the diffraction limit of a single aperture diameter $D \sim \lambda / D$, drops to $\lambda / B$, defined by the baseline $B$ separating the telescopes. Current baselines are on order of 100 to $200 \mathrm{~m}$, e.g. at the Very Large Telescope Interferometer (VLTI) operated by the European Southern Organisation (ESO), providing a potential improvement of one order of magnitude with respect to the resolution of an individual $8 \mathrm{~m}$ class telescope. The imaging resolution in the near infrared (NIR, 1 to $2.5 \mu \mathrm{m}$ ) becomes of order of a few milli-arcseconds. The potential astrometric performance, e.g. the measurement precision of the separation of a binary, is provided by the same geometric factor, scaled by the visibility and photometric signal to noise ratio (SNR), and can therefore reach the micro-arcsecond range, as is the goal of the PRIMA facility at VLTI. Some characteristics of its Fringe Sensor Unit, which contributed to setting up the concept described herein, are described in Gai et al. (2004).

In modern telescopes, the ideal flat wavefront from a remote star is partially recovered thanks to active and adaptive optics. The differential piston between telescopes, however, has to be directly measured at interferometric level; this sets a physical limitation to the duration of an elementary scientific exposure, and to the brightness of a source which could be measured with acceptable SNR before coherence degradation. The Fringe Tracker (FT) has the function of measuring in real time the optical path difference (OPD) perturbations on a bright source for on-line (hardware) or off-line (software) correction, thus allowing long scientific exposures. Moreover, it may correct additional disturbances introduced by the realistic limitations of the interferometer and of the environment. Several critical aspects are discussed in Basden \& Buscher (2005).

Current VLTI instruments provide interferometric measurements related to combination of either two or three telescopes at a time; this is less efficient than simultaneous 
combination of a larger number $N$ of apertures, also because of the operation overheads. The number of baselines grows more than linearly with $N$, thus providing significant advantages for new, multiple beam combiners. A promising concept for combination of four, six or eight beams, able to cope also with significant upgrades of VLTI, is proposed by an European team to ESO: the VLTI Spectro-Imager (VSI), described in Malbet et al. (2006). VSI aims at NIR measurements with good spectral and spatial resolution. Our study of a suitable Fringe Tracker, identified as a crucial contribution, has been endeavoured in parallel to development of the scientific beam combiner.

\section{The VSI Fringe Tracker Concept}

A key difference between a scientific $N$ beam combiner and a fringe tracker is that the latter does not require measurement on all baselines involved. Since any telescope is affected by an independent piston contribution, the problem's complexity (a number of unknowns) grows linearly with $N$. Using pair-wise combination, each beam is combined with only two other beams, thus providing $N$ complementary interferometric outputs which allow for the $N$ simultaneous phase estimates. This is actually a redundant measurement, since the interferometer is not usually sensitive to a common piston contribution, as in the case of a single telescope. The redundancy is minimal, as only one additional measurement is performed with respect to the minimum $N-1$ value. However, this improves the robustness at a system level, in particular with respect to infrastructure and environmental disturbances introducing occasional flux dropouts or large piston fluctuations in any of the beams. Such events could be identified on the appropriate beam, but the remaining telescopes may remain linked and stabilised with respect to each other, providing a graceful degradation during the disturbance and easier interferometer recovery when the nominal conditions are restored. Minimum redundancy thus provides better rejection of system noise.

The FT concept is based on few bulk optical components, simultaneously serving all (or most) of the $N$ beams; this ensures mutual alignment of the beams, with respect to both angular and longitudinal degrees of freedom, to within a fraction of wavelength, thanks to normal optical engineering tolerances. Such approach was used e.g. in laboratory demonstrators described in Ribak et al. (2007), with quite satisfactory results in easing the alignment and overall resiliency to disturbances. Bulk optics is a proven technique which can be extended efficiently to a combination of up to eight telescopes.

\section{Conclusions}

The VSI and FT concepts have been submitted to ESO and to national agencies for evaluation of the concept maturity and approval of the development plan.

\section{Acknowledgements}

We acknowledge the financial support of INAF - ref. 1478 (2005) for contributions to the FT lab prototype.

\section{References}

Malbet, F. et al. 2006, Proc. SPIE 6268, 62680Y

Basden, A. G. \& Buscher, D. F. 2005, MNRAS 357, 656

Gai, M. et al. 2004, Proc. SPIE 5491, 528

Ribak, E. N., Gai, M,, Loreggia, D., \& Lipson, S. G. 2007, Optics Letters 32, 1075 